\title{
Electron Microscopy of Yeast FACT Protein Complex
}

Karlova M.G. ${ }^{1}$, Valieva M.E. ${ }^{1,2}$, Klein Ya. ${ }^{1}$, Kozlova A.L. ${ }^{1}$, Studitsky V.M..${ }^{1,3 *}$ and Sokolova O.S. ${ }^{1 *}$

${ }^{1}$ Biology Faculty, Lomonosov Moscow State University, Moscow, Russia.

2. Max Planck Institute for Molecular Genetics, Berlin, Germany.

3. Fox Chase Cancer Center, Philadelphia, PA, USA.

*Corresponding authors: vasily.studitsky@fccc.edu, sokolova@mail.bio.msu.ru

DNA accessibility to regulatory proteins is essential for gene regulation; in chromatin, access to DNA is mediated by multiple protein factors, including chromatin remodelers and histone chaperones. FACT (facilitates chromatin transcription) is a histone chaperone that conducts large-scale ATP-independent nucleosome unfolding, that increases the accessibility of nucleosomal DNA [1]. FACT binding results in dramatic ATP-independent, symmetrical and reversible DNA uncoiling from the nucleosome, occurs without apparent loss of histones and proceeds via an 'all-or-none' mechanism [1], but the detailed mechanism of this process is unknown. FACT is involved in processes of transcription initiation and elongation, DNA replication and repair processes. FACT-dependent nucleosome unfolding modulates the accessibility of nucleosomal DNA, and this activity is an important function of FACT in vivo.

The structure and function of FACT are highly conserved in all eukaryotes, comprising of two subunits, SPT16 (Suppressor of Ty 16) and SSRP1 (Structure Specific Recognition Protein 1) in human, or Pob3 (Polymerase One Binding protein 3) in yeast.The main difference between the structures of human and yeast FACT is that the DNA-binding HMGB-domain is part of the human protein SSRP1, but, in yeast, this domain is represented by a separate protein Nhp6 (Non Histone Protein 6), which binds the DNA in a sequence-independent manner. Various domains of FACT subunits can interact with various individual components of nucleosomes (DNA, H3/H4 tetramer and $\mathrm{H} 2 \mathrm{~A} / \mathrm{H} 2 \mathrm{~B}$ dimers) [2]. We have proposed that these multiple interactions drive the highly cooperative nucleosome unfolding by FACT [1].

We evaluated the possibility that the unfolding of FACT can contribute to the nucleosome unfolding, using single particle electron microscopy. For this purpose, we studied the conformational diversity of purified FACT particles. The FACT complex was applied to hydrophilized copper EM grids and negatively stained with $1 \%$ uranyl acetate. Micrographs were acquired using an analytical transmission electron microscope Jem 2100 at $200 \mathrm{kV}$ with a magnification of $\mathrm{x} 40000$. Individual particles from the micrographs were selected in Eman 2.12 Boxer [3] with 100x100 pixel boxes. Particle sets were exported to Relion2.0.5 [4] for 2D classification and analysis.

Here, we demonstrated that, in the absence of nucleosomes, FACT, in solution, exists as a three-domain protein complex that exists in multiple "open" and "closed" conformations (Fig. 1A). During the transitions between these conformatios, positioning of the three domains, relative to each other, is changed. Comparison of electron densities within different classes of complexes suggests that Nhp6 binds to the terminal domains of the FACT (Fig. 1B). In the presence of the Nhp6 protein, the equilibrium is shifted towards more "open" conformations of FACT, suggesting that Nhp6 induces FACT unfolding in the absence of nucleosomes (Table 1). Since the Nhp6 protein is the critical player in FACT-dependent nucleosome unfolding, the data suggest that Nhp6-induced FACT unfolding likely participate in nucleosome unfolding by FACT [5]. 
References:

[1] ME Valieva et. al., Nat Struct Mol Biol 23 (2016), p. 1111.

[2] K Gurova et al., BBA - Gene regulatory Mechanism 1861 (2018), p. 892.

[3] G Tang et al., J Struct Biol. 157 (2007), p. 38.

[4] SH Scheres et al. J Mol Biol. 348 (2005), p. 139.

[5] The authors acknowledge funding from the RSF (19-74-30003). The JEOL2100 electron microscope is part of the User facility center of MSU "Electron microscopy in life sciences". All authors contributed equally to this paper.

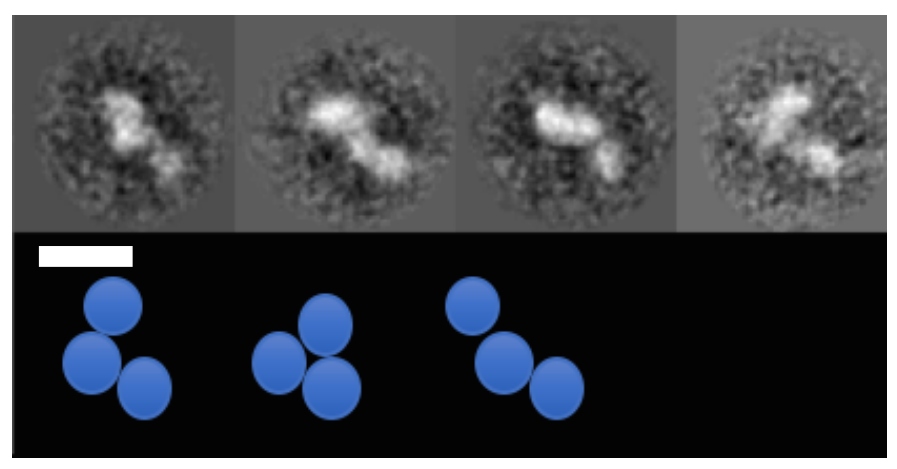

\section{Spt16/Pob3+Nhp6}

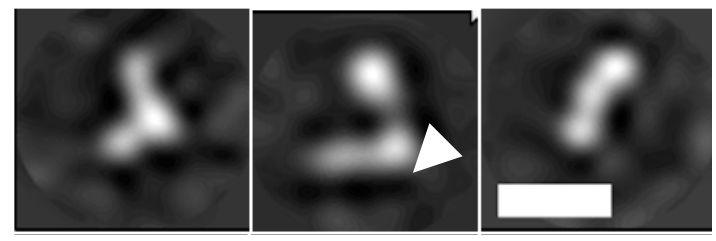

Figure 1. Electron microscopy of yFACT. (A) Spt16/Pob3 alone exists as a three-domain protein complex in different stages of being in a balance between "open" and "closed" conformations. (B) Schematic of conformational variability of Spt16/Pob3. (C) The Nhp6 subunit (arrow) adds an extravolume to one of the domains. Bars $-10 \mathrm{~nm}$.

\begin{tabular}{|c|c|c|c|c|c|}
\hline \multirow{2}{*}{ Sample } & \multicolumn{3}{|c|}{$\%$ of particles with corresponding angle } & \multirow{2}{*}{$\mathrm{N}$} \\
\cline { 2 - 5 } & $90^{\circ}$ & $180^{\circ}$ & $>90^{\circ}$ & $<90^{\circ}$ (closed) & \\
\hline $\begin{array}{c}\text { Spt16/Pob3+ } \\
\text { Nhp6 }\end{array}$ & 35,2 & 15,0 & 38,6 & 11,2 & 827 \\
\hline Spt16/Pob3 & 32,5 & 14,4 & 17,2 & 35,9 & 2090 \\
\hline
\end{tabular}

Table 1. The distribution of conformation of FACT with and without the Nhp6 subunit. $\mathrm{N}$ - number of analyzed particles. 